\title{
Prática Deliberada em Ciclos Rápidos: uma estratégia moderna de simulação
}

\author{
Rapid Cycle Deliberate Practice: a modern simulation strategy
}

\author{
Leandro Teixeira de Castro ${ }^{1}$, Thomaz Bittencourt Couto ${ }^{1} \bowtie$ \\ ${ }^{1}$ Centro de Simulação Realística, Instituto Israelita de Ensino e Pesquisa Albert Einstein. São Paulo, SP.
}

Como citar este artigo (How to cite this article):

Castro LD, Couto TB. Prática Deliberada em Ciclos Rápidos: uma estratégia moderna de simulação (Rapid Cycle Deliberate Practice: a modern simulation strategy). Sci Med. 2018;28(1):ID28849. http://doi.org/10.15448/1980-6108.2018.1.28849

\section{RESUMO}

OBJETIVOS: Descrever, por meio de revisão da literatura, a estrutura, efetividade, vantagens e desvantagens de uma nova estratégia de simulação clínica denominada Prática Deliberada em Ciclos Rápidos.

MÉTODOS: A revisão da literatura ocorreu entre junho e setembro de 2017. Foram procurados os termos "Prática Deliberada em Ciclos Rápidos", "Prática Deliberada" e "Ciclo Rápido", e seus termos equivalentes em espanhol e inglês, com filtro para estudos em seres humanos e para artigos publicados de janeiro de 2014 a agosto de 2017, nas bases de dados MEDLINE/PubMed, Web of Science, Scopus e SciELO.

RESULTADOS: Dos 264 artigos localizados a partir dos termos pesquisados, quatro estudos foram selecionados para esta revisão. Devido à pequena quantidade de estudos e à heterogeneidade dos grupos e dos desfechos medidos, optou-se por fazer uma revisão narrativa. A Prática Deliberada em Ciclos Rápidos consiste em simular casos clínicos em ciclos progressivamente mais complexos, repetindo-os até a aquisição da competência desejada pela equipe treinada; quando os objetivos de cada ciclo são alcançados, um novo ciclo se inicia, com incremento na dificuldade das tarefas exigidas. O método oferece múltiplas oportunidades de praticar as competências, automatização de habilidades e feedback direcionado. Apresenta uma série de vantagens sobre a simulação tradicional, incluindo aumento da satisfação dos participantes e melhoria na performance de desfechos-chave em ambiente de ressuscitação cardiopulmonar. Apresenta algumas desvantagens: discussão limitada de casos mais complexos ou cenários comportamentais e dificuldade do controle do tempo de cada ciclo, que depende do desempenho da equipe. CONCLUSÕES: Na Prática Deliberada em Ciclos Rápidos, participantes atuam em cenário de simulação com repetição de tarefas até a maestria na aquisição das competências desejadas. Esta estratégia apoia-se em técnicas consagradas, como a aprendizagem de mestria e a prática deliberada. Apesar de ter algumas desvantagens, como discussão limitada de cenários complexos ou comportamentais e dificuldade no controle do tempo, com um planejamento adequado e um bom treinamento de facilitadores, esse método pode ser uma excelente ferramenta para potencializar o aprendizado com simulação.

DESCRITORES: simulação; treinamento por simulação; educação médica; aprendizagem.

\section{ABSTRACT}

AIMS: To describe, through a literature review, the structure, effectiveness, advantages and disadvantages of a novel strategy in simulationbased training named Rapid Cycle Deliberate Practice.

METHODS: The literature review was conducted from June to September 2017. We searched for the terms "Rapid Cycle Deliberate Practice", "Deliberate Practice", "Rapid Cycle", and equivalent terms in Spanish and Portuguese, filtered for studies in human beings and for publications from January 2014 (when the term was coined) to August 2017, in the following databases: MEDLINE, Web of Science, Scopus and Scielo. RESULTS: Of the 264 articles found from the selected terms in our initial research, four studies were selected for this review. In Rapid Cycle Deliberate Practice, clinical cases are simulated in cycles progressively more complex, repeated until the acquisition of skills by the team of participants; when the objectives are reached, a new cycle begins, with more difficult tasks needed. This method offers multiple opportunities for skill practice, automation of skills and direct feedback. It presents a series of advantages over traditional simulation, like higher participants' satisfaction and better performance in key-outcomes in cardiopulmonary arrest scenarios. It has some disadvantages: limited discussion of complex or behavioral scenarios and difficult time control of each cycle, which are limited by team performance.

CONCLUSIONS: In Rapid Cycle Deliberate Practice, teams participate in a simulation scenario with repetitive practice until mastery of desired skills. This strategy leans on established techniques like mastery learning and deliberated practice. While having some disadvantages, like limited discussion of complex or behavioral scenarios and difficult time control, with proper planning and a good training of facilitators this method may be an excellent tool to increase learning with simulation.

KEYWORDS: simulation; simulation training; medical education; learning. 
Abreviaturas: BLS, Basic Life Support; PALS, Pediatric Advanced Life Support; PDCA, plan-do-check-act; PDCR, Prática Deliberada em Ciclos Rápidos; STAT, Simulation Team Assessment Tool.

\section{INTRODUÇÃO}

A metodologia tradicional de simulação clínica consiste no desenvolvimento de um caso clínico completo por um grupo de participantes, seguido pelo debriefing (reflexão e discussão) do caso com um facilitador [1]. Após a discussão dos pontos positivos e oportunidades de melhoria durante o debriefing, a simulação é encerrada sem que os participantes tenham a oportunidade de praticar novamente as tarefas exigidas no cenário. Esse modelo, com maior ênfase no processo de debriefing, pode ser ideal para a discussão crítica de casos com múltiplas oportunidades de desfechos - como casos comportamentais ou situações em que mais de uma conduta pode ser considerada correta para determinada situação clínica [1]. Mas também traz uma série de limitações importantes, entre elas a impossibilidade de os participantes praticarem novamente o cenário após receberem o feedback e o fato de que o tempo de debriefing acaba reduzindo o espaço para a prática, sendo muitas vezes superior ao tempo alocado para o desenvolvimento do cenário pelos participantes [2]. Em muitos casos, apenas alguns participantes do grupo desempenham papel ativo na simulação, levando os demais a adotarem a postura de observadores do cenário, com pouco estímulo para se engajarem ativamente durante o debriefing [3]. A dinâmica desse modelo de simulação contrasta com revisão sistemática da literatura de Issenberg et al. [4], que mostraram que os fatores considerados mais importantes para o bom desempenho em estudos de simulação clínica envolvem feedback de qualidade e prática repetida de habilidades.

Em 2009, Hunt et al. [5] publicaram estudo observacional evidenciando a falha de residentes de Pediatria em realizarem tarefas críticas durante uma ressuscitação pediátrica, mesmo após passarem por treinamento de simulação em suporte básico de vida (BLS - Basic Life Support) e suporte avançado de vida em pediatria (PALS - Pediatric Advanced Life Support). Em um estudo que abordou um caso simulado de ressuscitação pediátrica, apenas um terço dos residentes iniciou manobras de compressão torácica após o reconhecimento da parada cardiorrespiratória, e menos de metade deles realizou a desfibrilação em menos de dois minutos após o reconhecimento de uma parada cardiorrespiratória em taquicardia ventricular [5].

Em revisão da literatura publicada em 2008, Ericsson [6] descreve que a maestria no desempenho clínico não é atingida automaticamente através da experiência clínica, mas sim a partir da integração de um sistema complexo de ações para execução, monitoramento, planejamento e análise de performance; o estudo sugere que a forma mais efetiva para atingir maestria na performance seria através da prática deliberada. Este conceito de prática deliberada já é utilizado por profissionais de diversas outras áreas, como na música e em esportes de alto desempenho, com o objetivo de maximização de performance [7].

Com base nos estudos de Ericsson et al. [6], Hunt [2] cunhou em 2014 o termo Prática Deliberada em Ciclos Rápidos (PDCR), descrevendo uma nova estratégia de simulação. Nessa dinâmica, o mesmo caso clínico é simulado por diversas vezes até a aquisição da competência desejada por parte da equipe que está sendo treinada; quando os objetivos desse ciclo são alcançados, um novo ciclo se inicia, com incremento na complexidade das tarefas exigidas. A Figura 1, adaptada do artigo original de Hunt, resume essa dinâmica de treinamento [2]. Os três princípios básicos da PDCR consistem em: maximizar o tempo em que os alunos participam ativamente da prática deliberada, alocando de forma mais adequada o recurso da simulação aos objetivos de aprendizado; oferecer múltiplas oportunidades de praticar as competências de

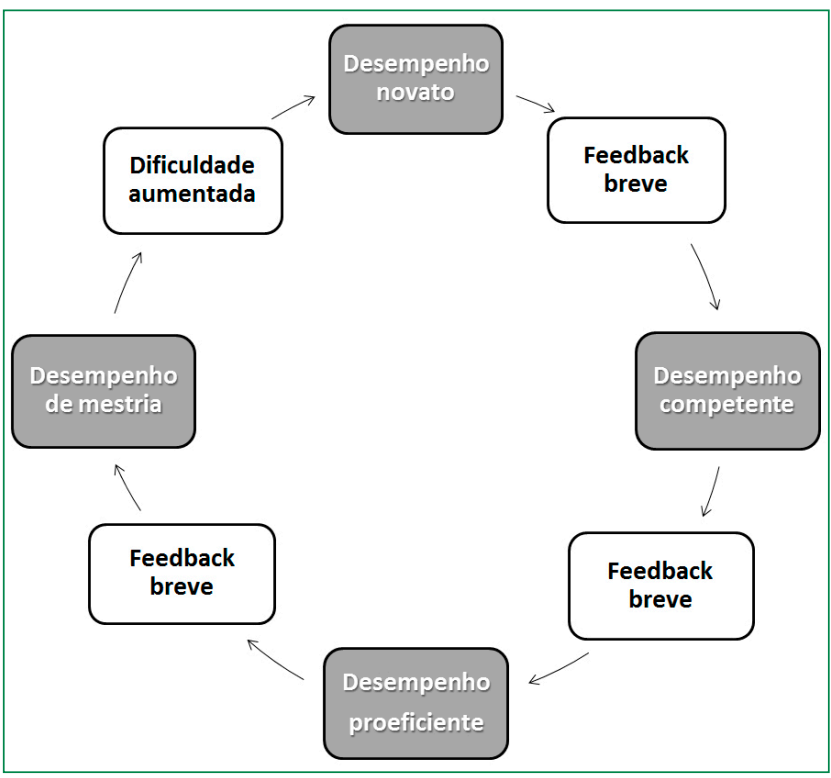

Figura 1. Representação gráfica da Prática Deliberada em Ciclos Rápidos. Adaptada de Hunt et al. [2]. 
forma correta, aplicando o conceito de automatização e "overlearning" (ou sobreaprendizagem), em que o aluno segue praticando a habilidade mesmo após demonstrar sua capacidade em realizar as tarefas de forma correta; por último, criar um ambiente de segurança psicológica, para permitir que os alunos aceitem o feedback direcionado sem adotarem uma postura defensiva. Assim, a Prática Deliberada em Ciclos Rápidos utiliza os conceitos de "Simulationbased mastery learning" (SBML), ou aprendizagem de mestria, estratégia já amplamente validada na literatura, baseada na necessidade de demonstração da proficiência do aluno em uma competência antes de passar para o próximo objetivo educacional e de um objetivo de treinamento que excede a proficiência, mirando o completo domínio, ou mestria em uma determinada competência $[2,8,9]$.

Até onde pudemos encontrar, não existem relatos do uso do método de PDCR em países de língua portuguesa ou espanhola. O presente estudo teve o objetivo de revisar a literatura disponível sobre o método de PDCR para ensino de profissionais de saúde e compará-lo à estratégia de simulação tradicional quanto ao desempenho posterior dos alunos em simulações ou casos reais após esse treinamento.

\section{MÉTODOS}

A revisão de literatura ocorreu entre julho e setembro de 2017. Por ser um método de descrição recente, com a primeira publicação no tema em 2014, a estratégia utilizada foi procurar os termos "Prática Deliberada em Ciclos Rápidos", "Prática Deliberada" e "Ciclo Rápido", e seus termos equivalentes em espanhol e inglês, com filtro para estudos em seres humanos e para artigos publicados de janeiro de 2014 a agosto de 2017, nas bases de dados MEDLINE/ PubMed, Web of Science, Scopus e SciELO.

Ambos os autores triaram os artigos selecionados pelo título e resumo e, caso necessário, revisaram artigos em sua versão integral. O critério de inclusão considerou qualquer desenho de estudo exceto revisões, envolvendo estudantes de graduação em cursos de saúde ou profissionais de saúde, submetidos ao método de PDCR comparado com simulação tradicional ou com nenhuma intervenção. Razões frequentes para excluir artigos foram trabalhos que não eram relacionados a treinamento em saúde, que usavam prática deliberada em outra forma que não ciclos rápidos ou que utilizavam ciclos rápidos para melhoria de qualidade, ou PDCA (plan-docheck-act).

\section{RESULTADOS DA SELEÇÃO}

Foram localizados 264 artigos a partir dos termos pesquisados, nenhum nos idiomas português ou espanhol. A partir da revisão dos artigos foram encontrados quatro estudos pertinentes para esta revisão. O diagrama de seleção de estudos está representado na Figura 2. Tendo em vista a pequena quantidade de estudos e a heterogeneidade dos grupos treinados e dos desfechos medidos, optou-se por fazer uma revisão narrativa sobre o tema.

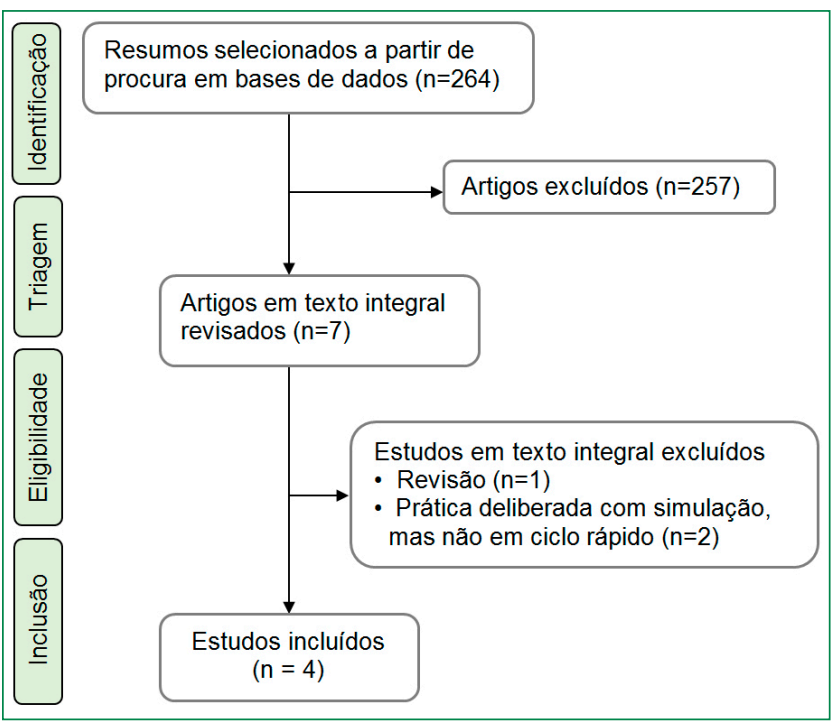

Figura 2. Fluxograma do processo de seleção dos estudos sobre Prática Deliberada em Ciclos Rápidos para esta revisão.

\section{CONTEÚDO DA REVISÃO}

\section{Efetividade da estratégia Prática Deliberada em Ciclos Rápidos}

Em 2014, estudo de pré e pós-intervenção com o uso da PDCR para um grupo de residentes de pediatria em cenário de reanimação cardiopulmonar evidenciou que o treinamento com esse método levou uma maior proporção de participantes a realizarem reanimação cardiopulmonar em menos de um minuto do diagnóstico da parada cardiorrespiratória $(71 \%$ vs $34 \%, \mathrm{p}<0,001)$, além de reduzir a pausa pré-choque após o diagnóstico de fibrilação ventricular (mediana de 27 vs 51 segundos, p<0,002) [2]. Em 2016, outro estudo em ambiente de ressuscitação pediátrica comparando a simulação tradicional com a estratégia PDCR encontrou tendência de maior aumento do desempenho de equipe utilizando a ferramenta 
STAT (Simulation Team Assessment Tool), sendo essa diferença estatisticamente significativa na comparação do domínio "fatores humanos" [10].

Há um único estudo, de 2015, utilizando PDCR para treinamento contínuo de enfermeiros, em oposição ao treinamento tradicional com simulação. Nesse estudo houve melhor satisfação com atividade e desempenho nos primeiros cinco minutos de ressuscitação cardiopulmonar após uso do método [11].

Em 2017, um estudo randomizado comparou dois métodos de simulação em suporte básico de vida: estratégia padrão (BLS - Basic Life Support), preconizada pela American Heart Association e estratégia de reanimação intra-hospitalar utilizando o método PDCR. Nesse estudo, os participantes que foram treinados utilizando o método PDCR iniciaram compressões torácicas em um tempo significativamente menor que os participantes treinados com o método tradicional, além de passarem mais tempo comprimindo o tórax e executando tarefas específicas da reanimação intra-hospitalar (uso de prancha de reanimação, conexão de fonte de oxigênio ao dispositivo bolsavalva-máscara e técnica adequada para ventilação difícil, entre outros) [12].

Ainda há um hiato na literatura em relação à eficácia deste método em cenários fora do ambiente de reanimação cardiopulmonar, principalmente em pacientes adultos.

\section{Preparação para a implementação do método}

A implementação da estratégia PDCR em cenários de simulação exige adequado planejamento e adaptação no estilo do facilitador, que terá papel muito mais ativo do que na simulação tradicional. Os passos a seguir devem ser analisados de forma criteriosa:

Referência bibliográfica: deve ser de ampla aceitação e previamente informada aos participantes, pois será a base do feedback dado no cenário; o facilitador deve se ater às informações da referência bibliográfica para manter a coerência do feedback. A bibliografia também serve para direcionar o aluno posteriormente, caso o mesmo apresente dúvidas conceituais que demorariam tempo demais para ser respondidas durante o cenário.

Planejamento das competências de cada ciclo: cada competência deve ser dividida em uma sequência de ações críticas que podem ser avaliadas de forma objetiva pelo facilitador do cenário. A realização das tarefas de um ciclo de forma adequada e na sequência correta autoriza os participantes a evoluírem para o próximo ciclo.
Capacitação dos facilitadores: é essencial que os facilitadores sejam orientados sobre os objetivos do cenário e os detalhes da estratégia PDCR, pois eles devem exercer papel mais ativo do que em outras técnicas para conduzir a simulação. $\mathrm{O}$ tempo permitido para os participantes praticarem deve ser balanceado com a intervenção durante erros em ações críticas; a intervenção deve ser executada de forma a reduzir ao máximo a interrupção no fluxo do cenário e assegurando um ambiente que não retalie o erro. O uso de frases ligadas a ações (como: não sinto pulso/iniciar compressões) demonstrou ser uma forma rápida de feedback que aumenta rapidamente a eficácia das habilidades técnicas em casos simulados de reanimação cardiopulmonar [13]. Outras formas de utilização do feedback, debriefing e microdebriefing (reflexão e discussão sucintas sem grande interrupção do cenário) foram publicadas recentemente [14].

Gestão do tempo do cenário: na estratégia PDCR não há um tempo previamente definido para a duração de cada ciclo; os participantes só avançam no cenário após demonstrarem maestria no cumprimento de todas as tarefas exigidas no ciclo que estão desenvolvendo. Esta dinâmica gera um desafio na gestão do tempo para os facilitadores, que devem estar preparados para "abandonar" os ciclos mais complexos caso haja muitas dificuldades por parte dos participantes em demonstrar maestria nas ações mais básicas.

Realização de um piloto: essa etapa, aconselhável no planejamento de qualquer cenário de simulação, torna-se ainda mais essencial em um cenário de PDCR. Durante o piloto podem ser identificados problemas na gestão do tempo de cada ciclo e momentos críticos do desempenho da equipe naquele cenário. Além disso, a programação do simulador e eventualmente o papel de um paciente padronizado podem ser ajustados de modo a aperfeiçoar o cenário.

Há na literatura outros artigos explicitando o delineamento da implementação da estratégia PDCR, com poucas modificações em relação à estratégia aqui descrita, mas com maior enfoque em aspectos do treinamento de ressuscitação pediátrica para médicos residentes $[2,15]$.

\section{Desvantagens do método}

Embora ofereça diversas vantagens em relação à simulação tradicional, o método PDCR apresenta algumas desvantagens. Como o feedback em um cenário PDCR é direcionado e objetivo, há pouco espaço para reflexão dos participantes [15]. Métodos de debriefing baseados no inquérito e desenvolvimento de discussão 
crítica com os participantes, já consagrados na literatura [16] não podem ser plenamente utilizados na estratégia PDCR, por interromperem demasiadamente o tempo planejado para a prática. Outro grande desafio é quando a simulação envolve casos com diversas possibilidades de conduta igualmente corretas para o mesmo cenário; a experiência da simulação pode se tornar inconsistente ao longo dos ciclos, impactando negativamente a efetividade do treinamento - este é o caso dos cenários comportamentais, que podem ser mais bem explorados com uso de outras estratégias de simulação. Por fim, o tempo para a realização das tarefas dentro de um ciclo não pode ser estritamente controlado, pois depende da demonstração de maestria pelos participantes; em uma equipe muito heterogênea, pode ser necessário abandonar um ciclo mais complexo para garantir a maestria das habilidades mais básicas por todos os participantes, dificultando a padronização do treinamento entre equipes.

\section{CONCLUSÕES}

O método de PDCR traz vantagens importantes em relação à simulação tradicional, incluindo a maximização do tempo de prática no centro de simulação; priorizando a alocação de recursos para o método mais adequado ao incremento de habilidades; expondo continuadamente todos os participantes ao cenário de simulação, com repetição de tarefas até a maestria na aquisição das competências desejadas; e criando um ambiente seguro que utiliza o erro como ferramenta de aprendizagem. Esta estratégia se apoia em técnicas já amplamente validadas na literatura, como a aprendizagem de mestria e o ganho de performance através da ênfase na prática deliberada.

A utilização do método pode não ser adequada em cenários comportamentais ou com múltiplas possibilidades de conduta. Entretanto, com um bom planejamento e adequado treinamento dos facilitadores, a estratégia PDCR pode ser uma excelente ferramenta para potencializar o aprendizado com simulação.

\section{NOTAS}

Apoio financeiro

A execução desta revisão recebeu apoio financeiro do Centro de Simulação Realística do Instituto Israelita de Ensino e Pesquisa Albert Einstein.

Declaração de conflito de interesses

Os autores declaram não haver conflitos de interesses relevantes ao conteúdo deste estudo.

\section{Contribuições dos autores}

Todos os autores fizeram contribuições substanciais para concepção, ou delineamento, ou aquisição, ou análise ou interpretação de dados; e redação do trabalho ou revisão crítica; e aprovação final da versão para publicação.

\section{REFERÊNCIAS}

1. Sawyer T, Eppich W, Brett-Fleegler M, Grant V, Cheng A. More Than One Way to Debrief: A Critical Review of Healthcare Simulation Debriefing Methods. Simul Healthc. 2016;11(3):209-17. https://doi.org/10.1097/SIH.0000000000000148

2. Hunt EA, Duval-Arnould JM, Nelson-McMillan KL, Bradshaw JH, Diener-West M, Perretta JS, Shilkofski NA. Pediatric resident resuscitation skills improve after "rapid cycle deliberate practice" training. Resuscitation. 2014;85(7):945-51. https://doi.org/10.1016/j.resuscitation.2014.02.025

3. Quilici AP, Bicudo AM, Gianotto-Oliveira R, Timerman S, Gutierrez F, Abrão KC. Faculty perceptions of simulation programs in healthcare education. Int J Med Educ. 2015;6:166-71. https://doi.org/10.5116/ijme.5641.0dc7

4. Issenberg SB, McGaghie WC, Petrusa ER, Lee Gordon D, Scalese RJ. Features and uses of high-fidelity medical simulations that lead to effective learning: a BEME systematic review. Med Teach. 2005;27(1):10-28. https://doi. org/10.1080/01421590500046924

5. Hunt EA, Vera K, Diener-West M, Haggerty JA, Nelson KL, Shaffner DH, Pronovost PJ. Delays and errors in cardiopulmonary resuscitation and defibrillation by pediatric residents during simulated cardiopulmonary arrests. Resuscitation. 2009;80(7):819-25. https://doi.org/10.1016/j.resuscitation.2009.03.020

6. Ericsson KA. Deliberate practice and acquisition of expert performance: a general overview. Acad Emerg Med. 2008;15(11):988-94. https://doi.org/10.1111/j.1553-2712.2008.00227.x

7. Ericsson KA, Krampe RT, Tesch-Römer C. The role of deliberate practice in the acquisition of expert performance. Psychol Rev. 1993; 100:363-406. https://doi.org/10.1037/0033-295X.100.3.363

8. Driskell JE, Willis RP, Copper C. Effect of overlearning on retention. J Appl Psychol, 1992;77(5):615-22. https://doi. org/10.1037/0021-9010.77.5.615

9. Cook DA, Brydges R, Zendejas B, Hamstra SJ, Hatala R. Mastery learning for health professionals using technologyenhanced simulation: a systematic review and meta-analysis. Acad Med 2013;88:1178-86. https://doi.org/10.1097/ ACM.0b013e31829a365d 
10. Lemke DS, Fielder EK, Hsu DC, Doughty CB. Improved Team Performance During Pediatric Resuscitations After Rapid Cycle Deliberate Practice Compared With Traditional Debriefing: A Pilot Study. Pediatr Emerg Care. Pediatric Emergency Care; 2016 Oct 6. https://doi.org/10.1097/PEC.0000000000000940

11. Kutzin J, Janicke P. Incorporating Rapid Cycle Deliberate Practice Into Nursing Staff Continuing Professional Development. J Contin Educ Nurs. 2015;46(7) 299-301. https://doi.org/10.3928/00220124-20150619-14

12. Hunt EA, Duval-Arnould JM, Chime NO, Jones K, Rosen M, Hollingsworth M, Aksamit D, Camacho C, Nogee DP, Jung J, Nelson-McMillan K, Shilkofski N, Peretta JS. Integration of in-hospital cardiac arrest contextual curriculum into a basic life support course: a randomized, controlled simulation study. Resuscitation. 2017;114:127-32. https://doi. org/10.1016/j.resuscitation.2017.03.014

13. Hunt EA, Cruz-Eng H, Bradshaw JH, Hodge M, Bortner T, Mulvey CL, McMillan KN, Duval-Arnould JM, Jones K, Shilkofski NA, Rodgers DL, Sinz EH. A novel approach to life support training using "action-linked phrases". Resuscitation. 2015;86:1-5. https://doi.org/10.1016/j.resuscitation.2014.10.007

14. Eppich WJ, Hunt EA, Duval-Arnould JM, Siddall VJ, Cheng A. Structuring Feedback and Debriefing to Achieve Mastery Learning Goals. Academic Medicine. 2015;90(11):1501-8. https://doi.org/10.1097/ACM.0000000000000934

15. Doughty C, Welch-Horan T, Hsu D, Fielder E, Pereira F, Little-Wienert K, Kaziny B, McManemy J, Lemke D. Rapid Cycle Deliberate Practice Pediatric Simulation Scenarios. MedEdPORTAL. 2015;11:10134. https://doi.org/10.15766/ mep_2374-8265.10134

16. Jaye P, Thomas L, Reedy G. "The Diamond": a structure for simulation debrief. The Clinical Teacher. 2015;12(3):171-5. https://doi.org/10.1111/tct.12300 